\title{
Key Processes in Solar-Terrestrial Physics
}

\author{
Walter D. Gonzalez • James L. Burch
}

Published online: 29 April 2011

(C) Springer Science+Business Media B.V. 2011

During the week of October 4-9, 2009, about 160 participants from 19 countries met at the Itamambuca resort area of Ubatuba, Sao Paulo, Brazil to discuss the influence of solar variability on geophysical and heliospheric phenomena at a conference organized by the International Living With a Star (ILWS) Program of NASA and by the National Institute of Space Research (INPE) of Brazil.

Five of the invited review papers of this Conference appear in this special issue along with an additional paper on magnetospheric reconnection by F.S. Mozer and P.L. Pritchett, which was invited to cover a missing important subject within the solar-terrestrial physics domain.

One of the review papers of this issue (by H. Hudson, 10.1007/s11214-010-9721-4) deals with a key topic in solar physics dedicated to the "Global properties of solar flares", in which the author discusses general flare energetics, radiation, mass loss in CMEs and global wave disturbances. The latter involve Type II radio burst exciters, Moreton waves, the EIT waves and "sunquake" acoustic waves. The author notes that the impulsive phase of flares dominates the flare energetics and these global manifestations. He also shows that the momentum and energy in the impulsive phase largely reside in the field and not in the observable plasma.

Two review papers are dedicated to the interplay of the interplanetary medium and the magnetosphere of the Earth. One of those (by A.T.Y. Lui, 10.1007/s11214-011-9751-6) reviews the "Grad-Shafranov reconstruction of magnetic flux ropes in the near-Earth space", in which the author discusses some properties of magnetic flux ropes (MFR) and their role in the interplanetary and magnetospheric plasma dynamics. For MFRs that are $2 \mathrm{D}$ equilibrium structures, the G-S reconstruction technique can be used to reveal 2D maps of associated plasma parameters and is applied to study events in the solar wind, the Earth's magnetopause

W.D. Gonzalez

National Institute of Space Research, S. J. dos Campos, Brazil

J.L. Burch (凶)

Southwest Research Institute, San Antonio, TX, USA

e-mail: jburch@swri.edu 
and magnetotail. For these events the author presents a range of estimated total field-aligned current and total magnetic flux amplitudes associated with the MFR structures. The other paper (by W.D. Gonzalez et al., 10.1007/s11214-010-9715-2) presents a review of the "Interplanetary origin of intense, superintense and extreme geomagnetic storms." In this paper the authors review the interplanetary causes of intense (Dst $<-100 \mathrm{nT}$ ) and superintense (Dst $<-250 \mathrm{nT}$ ) storms as discussed in the literature mainly for solar cycle 23. On extreme storms (Dst $<-400 \mathrm{nT}$ ), the paper deals with the related modest interplanetary observations collected during the space era and concentarates later on the historical storms studied from ground magnetic records since 1842. Among them, the famous Carrington extreme storm of September 2, 1859 is discussed together with the "failed" Carrington-type storm of August 4,1972 . Further, by studying the occurrence of historical extreme storms in the context of the Gleissberg modulation/cycle of solar activity, the authors discuss the once/century occurrence behavior of Carrington-type extreme storms.

In magnetospheric physics two review papers are presented. The first paper (by V. Vasyliūnas, 10.1007/s11214-010-9696-1) deals with "Physics of magnetospheric variability," in which the author notes that presently many approaches to the study of magnetospheric processes are still based on balance conditions for quasi-stable equilibrium with limited applicability when dealing with time-variable phenomena and the determination of causeeffect relations. He proposes instead that large-scale variability of the magnetosphere, affected by solar-wind variability and non-equilibrium internal dynamics of the magnetosphere, should be studied with the full set of MHD equations supplemented by the unique constraints of the large-scale plasma, namely the requirement of charge quasi-neutrality constraining the electric field to be determined by the plasma dynamics and the electric currents to match the curl of the magnetic filed affected by the plasma stresses. This time-dependent approach to the study of magnetospheric dynamics is based on three interrelated processes: stress equilibrium and non-equilibrium, magnetic flux transport, and energy conversion and dissipation. The author finds that this approach provides a logical framework to study largescale magnetospheric phenomena such as storms and substorms.

The second magnetospheric paper (by F.S. Mozer and P.L. Pritchett, 10.1007/s11214010-9681-8) deals with "Electron physics of asymmetric magnetic field reconnection." In this review the authors deal with properties of asymmetric reconnection on electron scale lengths, involving parallel electric fields and electron acceleration. Based on observational identification of asymmetric reconnection at the Earth's magnetopause, supplemented by 2D particle-in-cell simulations, the authors show that in asymmetric reconnection many well known properties of symmetric reconnection are absent. They also discuss parallel electric fields, electron out-of-plane flows, the onset of electron acceleration at scales of about one to a few ion inertia lengths in the outflow direction, and high speed electron jets flowing away from a diffusion region extending many tens of ion inertia scale lengths. These studies are restricted by 2D limitations of collisionless reconnection models and by the ratio of only up to a few hundred between the ion and electron masses in the simulations.

For the ionosphere, a review by B. Fejer (10.1007/s11214-010-9690-7) about "Low latitude ionospheric electrodynamics" deals with the highly variable electrodynamic processes in the low latitude ionosphere for quiet and disturbed conditions. These processes are studied in the context of longitude-dependent climatology of electric fields and currents in response to the atmospheric and solar wind-magnetospheric driving processes. The author presents a comprehensive review of quiet and moderately disturbed conditions; however, for highly disturbed conditions, he notes that there is lack of present understanding of the several highly variable ionospheric phenomena affected by preconditioning processes and by the 
interaction of the prompt-penetration and disturbed dynamo processes. The author suggests that this lack of knowledge should be minimized by large-scale coordinated observational efforts and related computational modeling.

In our opinion, this set of review papers deals with a significant fraction of the important key phenomena in solar-terrestrial relations, but certainly many other important topics are still missing. Perhaps as a result of future ILWS Conferences these additional research topics in Solar-Terrestrial Physics can be treated in future special issues of Space Science Reviews. 